\title{
RAMAN LASER: MATHEMATICAL AND NUMERICAL ANALYSIS OF A MODEL
}

\author{
François Castella $^{1}$, Philippe Chartier ${ }^{2}$, Erwan Faou ${ }^{2}$, Dominique Bayart $^{3}$, \\ Florence LePlingard ${ }^{3}$ And CATHERINe Martinelli ${ }^{3}$
}

\begin{abstract}
In this paper we study a discrete Raman laser amplification model given as a LotkaVolterra system. We show that in an ideal situation, the equations can be written as a Poisson system with boundary conditions using a global change of coordinates. We address the questions of existence and uniqueness of a solution. We deduce numerical schemes for the approximation of the solution that have good stability.
\end{abstract}

Mathematics Subject Classification. 65L10,65L20.

Received: July 29, 2003.

\section{INTRODUCTION}

The problem described in this paper originates from a model of Raman laser amplification effect in an optic fiber $[6,7]$. Standard discrete models of this phenomenon $[1,8]$ lead to a system of differential equations of LotkaVolterra form (see for instance [5]), where high-frequency waves traveling forward and backward in the fiber disseminate part of their energy to low-frequency waves through a prey-predator process. Boundary conditions corresponding to Bragg reflecting lattices are imposed on both sides of the laser cavity [8].

In the case of an idealized fiber, this system turns out to have a Poisson structure (see for instance [5]) for which we can exhibit explicitly the Hamiltonian and the Casimir invariants. However, the underlying Hamiltonian function is affine with respect to the unknowns. The corresponding invariant manifold is thus not compact so that the existence of a solution remains a non-trivial question. Moreover, the system is posed as a boundary value problem. These aspects contribute to make a numerical approximation difficult to obtain: for instance, the shooting method [2] is to be banned here due to the presence of nonlinearities (most initial values would lead to blow-up in finite time); more elaborated methods, such as finite differences, collocation, or multiple shooting, are possible alternatives, but might become prohibitively costly in large dimension.

Another difficulty comes from the fact that in the original variables, there exists always a "trivial" solution corresponding to the case where the Raman amplification effect has not yet started. Numerically, the presence of this dummy solution makes the choice of the initial values in an iterative process difficult to determine.

\footnotetext{
Keywords and phrases. Optical device, Raman gain, Poisson system, integro-differential equations.

${ }^{1}$ IRMAR, Université de Rennes 1, Campus Beaulieu, 35042 Rennes Cedex, France. e-mail: Francois.Castella@univ-rennes1.fr

2 INRIA Rennes, Campus Beaulieu, 35042 Rennes Cedex, France. e-mail: Philippe.Chartier@irisa.fr; Erwan.Faou@irisa.fr

3 ALCATEL Research \& Innovation, Unité Transmissions Photoniques, Route de Nozay, 91460 Marcoussis, France.

e-mail: Dominique.Bayart@alcatel.fr; Florence.Leplingard@alcatel.fr; Catherine.Martinelli@alcatel.fr
} 
Here we prove that the Poisson system can be brought to canonical form through a global change of coordinates. Note that the change of coordinates defined in Darboux-Lie's Theorem is usually local and that the literature offers only few examples of such global transformations (see [5] p. 241 for a nice example). We show that for an ideal fiber the equations can be written as

$$
u^{\prime}=G \nabla_{u} H(u, d) \quad \text { with } \quad H(u, d)=\sum_{i=1}^{n} d_{i} \sinh u_{i}
$$

where $u$ is an $n$-dimensional unknown vector of functions defined on the fiber, $d$ an unknown element of $\mathbb{R}^{n}$, $G$ a skew-symmetric matrix and $H(u, d)$ the Hamiltonian of the problem. At this stage, getting a canonical Poisson system requires only to bring the constant skew-symmetric matrix $G$ to canonical form. Note that the $d_{i}$ 's are Casimir invariants of the underlying Poisson structure [5]. In this form, the "trivial" solution does not show up, but the problems of existence and uniqueness of the solution are still there. Another difficulty arises from the fact that the boundary conditions depend also on the unknown values of the Casimir invariants $d_{i}$. In the general case (i.e. not for an idealized fiber), we show that we can write the problem in a form close to (1) where the $d_{i}$ 's remain invariants of the problem with unknown values.

We show that it is actually possible to take benefit of the available free parameters $d$ so as to reformulate the problem as a Cauchy problem for a system of integro-differential equations. In this form, the problem is well-posed: using standard techniques (Schauder's theorem), the existence of solutions can be easily proved for boundary conditions independent of $d[3]$. Uniqueness for boundary values that are not too far apart and an arbitrary dimension is also shown. Note that ad hoc techniques allow for the treatment of the one and two-dimensional cases for arbitrary boundary values [3]. Eventually, we prove the existence and uniqueness of a solution to the original problem (with boundary conditions depending on $d$ ) under strong assumptions on the data.

Using the integro-differential formulation of the problem, we derive a numerical Picard-like scheme converging toward the solution under smallness assumptions on the data. We conclude this work by giving numerical examples showing that this scheme converges linearly to the solution in practical cases.

The paper is organized as follows: in Section 2, we describe the original Lotka-Volterra equations and in Section 3 we exhibit the Poisson structure in the case of an ideal fiber. We then show a global version of the Darboux-Lie Theorem for this system.

Sections 5-7 are devoted to the proof of existence and uniqueness results for the general problem using the change of unknowns defined in Section 4. In practical cases, the matrix $G$ is invertible when $n$ is even, and singular with a zero eigenvalue of multiplicity 1 when $n$ is odd. We thus distinguish these two cases. In Section 5 , we first consider the simplest case where $n$ is even, $G$ is invertible and the boundary conditions are independent of $d$. We combine this result with a fixed-point theorem to obtain an existence and uniqueness result for the general case (i.e. with boundary conditions depending on $d$ ) when $n$ is even in Section 6. Eventually, Section 7 deals with the case where $n$ is odd.

Finally we give numerical results in Section 8.

\section{A MODEL OF CASCADED RAMAN FiBER LASER}

We denote by $L$ the length of the cavity, and we suppose that $n$ rays at given frequencies $\nu_{1}, \nu_{2}, \ldots, \nu_{n}$ are represented by $n$ functions $F_{i}(x)$ and $B_{i}(x)$ for $x \in[0, L]$ denoting the powers of the forward and backward waves respectively.

The model equations can be written as follows, where the index $i$ runs from 0 to $n$ (see $[1,8]$ ):

$$
\begin{aligned}
F_{i}^{\prime} & =-\alpha_{i} F_{i}+\sum_{j<i} g_{i j}\left(F_{j}+B_{j}\right) F_{i}-\sum_{j>i} g_{i j}\left(F_{j}+B_{j}\right) F_{i} \\
B_{i}^{\prime} & =\alpha_{i} B_{i}-\sum_{j<i} g_{i j}\left(F_{j}+B_{j}\right) B_{i}+\sum_{j>i} g_{i j}\left(F_{j}+B_{j}\right) B_{i} .
\end{aligned}
$$


Here and in the sequel, the ${ }^{\prime}$ denotes the derivation with respect to $x \in[0, L]$. The coefficients $g_{i j}$ are non negative and represent the Raman gain between the wavelengths of levels $i$ and $j$. The coefficients $\alpha_{i}>0$ are attenuation coefficients. We define the Raman gain matrix $G=\left(G_{i j}\right)$ by:

$$
\begin{aligned}
& G_{i j}=-g_{i j} \quad \text { if } \quad j>i, \\
& G_{i j}=g_{i j} \quad \text { if } i<j, \\
& G_{i i}=0 .
\end{aligned}
$$

We can now rewrite equations (2) in a more compact Lotka-Volterra form as follows:

$$
\begin{aligned}
F_{i}^{\prime} & =-\alpha_{i} F_{i}+\sum_{j=1}^{n} G_{i j}\left(F_{j}+B_{j}\right) F_{i} \\
B_{i}^{\prime} & =\alpha_{i} B_{i}-\sum_{j=1}^{n} G_{i j}\left(F_{j}+B_{j}\right) B_{i} .
\end{aligned}
$$

To complete the description of the problem, it remains to consider the boundary conditions in 0 and $L$. They read

and

$$
F_{1}(0)=P \quad \text { and } \quad F_{i}(0)=R_{i}^{0} B_{i}(0), \quad i=2, \ldots, n
$$

$$
B_{i}(L)=R_{i}^{L} F_{i}(L), \quad i=1, \ldots, n-1 \quad \text { and } \quad B_{n}(L)=R_{\text {out }} F_{n}(L),
$$

where the coefficients $R_{i}^{0}$ and $R_{i}^{L}$ are reflectivity coefficients of the Bragg lattices in $x=0$ and $x=L$ respectively, and $R_{\text {out }}$ is the last reflectivity coefficient (see [8]). The number $P$ is given and represents the pump power injected in the cavity at the frequency $\nu_{1}$. We will mainly consider the situation where $R_{i} \simeq 1$ and $R_{\text {out }}<1$ (usually $R_{\text {out }} \simeq 0.15$ and $R_{i}^{0}=R_{i}^{L} \simeq 0.99$ ).

Note that the system (2-3-4) possesses the "trivial" solution

$$
F_{1}(x)=P \exp \left(-\alpha_{1} x\right), \quad B_{1}(x)=R_{1}^{L} P \exp \left(\alpha_{1}(x-2 L)\right)
$$

and $F_{i}=B_{i}=0$ for $i \geq 2$. This solution corresponds to the case where the Raman amplification effect has not yet appeared. Indeed, the system (2) describes a stationary regime of more general time-dependent equations. In practice, the laser starts on the noise due to a further term not present in equations (2-3-4), the so-called "Amplified Spontaneous Emission" (ASE) (see [6]). From a mathematical point of view, when the (ASE) term is taken into account, the only admissible stationary regime is the non-trivial one. However, as soon as the laser starts, the contribution of the (ASE) can be completely neglected. We are thus looking for a physical solution of (2-3-4), satisfying the further assumptions:

$$
F_{i}>0 \text { and } B_{i}>0 \text { for } i=1, \ldots, n
$$

Even at this early stage, it is interesting to notice that the system has several mathematical invariants. A simple calculation shows indeed that

$$
\forall i=1, \ldots, n, \quad \forall x \in[0, L], \quad\left(F_{i} B_{i}\right)(x)=\left(F_{i} B_{i}\right)(0)=\left(F_{i} B_{i}\right)(L) .
$$

If we make the further assumption that $G$ is skew-symmetric (that is to say that the exchange of energy is symmetric), and that the $\alpha_{i}$ 's are all vanishing (meaning that there is no absorption of energy within the fiber), then we can further notice that $\sum_{j}\left(F_{j}-B_{j}\right)$ is kept constant along the fiber. This quantity can be interpreted as the energy of the system and its preservation in absence of attenuation is physically sounded.

Remark 2.1. In practical cases, the matrix $G$ is close to a bidiagonal matrix $\tilde{G}$ such that $\tilde{G}_{i j}=0$ for $|i-j|>1$, $\tilde{G}_{i i}=0$ for $i=1, \ldots, n, \tilde{G}_{i, i+1}=-\sigma$ for $i=1, \ldots, n-1$ and $\tilde{G}_{i+1, i}=\sigma$ for $i=2, \ldots, n$, where $\sigma$ is a real positive number. Note that with this definition, $\tilde{G}$ is invertible when $n$ is even and singular with the eigenvalue 0 of multiplicity 1 when $n$ is odd. This corresponds to the case where we only take into account the interactions 
between successive frequencies, and where we suppose that the value of the Raman gain does not depend on the frequencies, but only on the difference between two frequencies (see [8]).

The existence of these invariants will become natural in the next section, where the system is shown to have a Poisson structure. It is a well-known fact that such systems can be brought back to canonical form, through a local change of variables. In the context of the present study, it is in fact possible to exhibit a global change of variables and this is the subject of Section 4 .

\section{A conservative model With Poisson structure}

We consider here a somewhat idealized model, which can be viewed as a simplified form of the previous one. The so-obtained system has obviously the advantage to be more tractable from a mathematical point of view and to give more insight. In this section, we thus make the following assumptions:

(1) $g_{i j}=-g_{j i}$, so that the matrix $G$ is skew-symmetric;

(2) the matrix $G$ is of maximal rank: $G$ is invertible if $n$ is even and $G$ is of rank $n-1$ is $n$ is odd;

(3) $\alpha_{i}=0$ for all $i=1, \ldots, n$ (see Rem. 2.1).

In the following we set $Y=(F, B) \in \mathbb{R}^{2 n}$ and we define $G(F, B)$ as being the $n \times n$ matrix with coefficients $G_{i j} F_{i} B_{j}$. The $2 n$-dimensional square-matrix $J(Y)$ is then constructed by the equation

$$
J(Y):=\left(\begin{array}{rr}
G(F, F) & -G(F, B) \\
-G(B, F) & G(B, B)
\end{array}\right)
$$

and is clearly skew-symmetric. We write $J_{\alpha \beta}(Y)$ the coefficients of this matrix $(\alpha, \beta=1, \ldots, 2 n)$. Now for two functions $H$ and $K$ of $Y$ we define the bracket

$$
\{H, K\}(Y)=\sum_{\alpha, \beta=1}^{2 n} \frac{\partial H(Y)}{\partial Y_{\alpha}} J_{\alpha \beta}(Y) \frac{\partial K(Y)}{\partial Y_{\beta}} .
$$

We will see that this defines a Poisson bracket, i.e. satisfies for all $H, K$ and $Q$ functions of $Y$ the three identities

$$
\begin{array}{ll}
\{H, K\}=-\{K, H\} & \text { (skew-symmetry) } \\
\{\{H, K\}, Q\}+\{\{K, Q\}, H\}+\{\{Q, H\}, K\}=0 & \text { (Jacobi identity) } \\
\{H \cdot K, Q\}=H \cdot\{K, Q\}+K \cdot\{H, Q\} & \text { (Leibniz rule) }
\end{array}
$$

This is a consequence of the following lemma (with $m=2 n$ ):

Lemma 3.1. Let $m \geq 1$ and $A_{\alpha \beta}$ be a skew-symmetric matrix of order $m\left(A_{\alpha \beta}=-A_{\beta \alpha}\right)$. Let $A(Y)$ be the matrix of order $m$ with coefficients $A_{\alpha \beta} Y_{\alpha} Y_{\beta}$. Then the application

$$
(H, K) \mapsto \sum_{\alpha, \beta=1}^{m} \frac{\partial H(Y)}{\partial Y_{\alpha}} A_{\alpha \beta}(Y) \frac{\partial K(Y)}{\partial Y_{\beta}}
$$

defines a Poisson Bracket.

Proof. It is well known (see [5]) that the result holds true if the matrix $A(Y)$ satisfies the relation

$$
\sum_{\alpha=1}^{m}\left(\frac{\partial A_{\beta \sigma}(Y)}{\partial Y_{\alpha}} A_{\alpha \delta}(Y)+\frac{\partial A_{\sigma \delta}(Y)}{\partial Y_{\alpha}} A_{\alpha \beta}(Y)+\frac{\partial A_{\delta \beta}(Y)}{\partial Y_{\alpha}} A_{\alpha \sigma}(Y)\right)=0
$$


for all $Y \in \mathbb{R}^{m}$. But we have

$$
\sum_{\alpha=1}^{m} \frac{\partial A_{\beta \sigma}(Y)}{\partial Y_{\alpha}} A_{\alpha \delta}(Y)=A_{\beta \sigma} A_{\beta \delta} Y_{\beta} Y_{\delta} Y_{\sigma}+A_{\beta \sigma} A_{\sigma \delta} Y_{\beta} Y_{\delta} Y_{\sigma}
$$

Thus the relation (8) is equivalent to

$$
A_{\beta \sigma} A_{\beta \delta}+A_{\beta \sigma} A_{\sigma \delta}+A_{\sigma \delta} A_{\sigma \beta}+A_{\sigma \delta} A_{\delta \beta}+A_{\delta \beta} A_{\delta \sigma}+A_{\delta \beta} A_{\beta \sigma}=0
$$

and we see that this relation is satisfied using the fact that $A_{\alpha \beta}$ is skew-symmetric.

Using the partition $Y=(F, B)$, we see that $J(Y)$ is of the form (6), so that $(7)$ defines a Poisson Bracket. A simple computation then yields the first part of the following result:

Proposition 3.2. Suppose that $\alpha_{i}=0$ for $i=1, \ldots, n$ and that $G$ is skew-symmetric of maximal rank. Then the system (2) is equivalent to the system

$$
Y^{\prime}=J(Y) \nabla H_{0}(Y)
$$

where $J(Y)$ is the matrix (6) and $H_{0}(Y)$ is the Hamiltonian

$$
H_{0}(Y)=\sum_{i=1}^{n}\left(F_{i}-B_{i}\right) \quad \text { for } \quad Y=(F, B) .
$$

Furthermore, the system possesses $n$ Casimir invariants $c_{i}=F_{i} B_{i}, i=1, \ldots, n$. If $n$ is odd, it has the additional invariant $\sum_{i=1}^{n} a_{i} \log F_{i}$ where $a=\left(a_{i}\right)_{i=1}^{n}$ is such that $a^{T} G=0$.

Proof. By construction, if $Y_{\sigma} \neq 0$ for $k=1, \ldots, 2 n$, the matrix $J(Y)$ defined in (6) has the same rank as the matrix $G$. Moreover, if we set $c_{i}=F_{i} B_{i}$ for $i=1, \ldots, n$, we see that the vectors $\nabla c_{i}$ are in the kernel of $J(Y)$. As these vectors are linearly independent, the $c_{i}$ 's are $n$ Casimir of the system. If $n$ is even, there is no other Casimir since the rank of $J(Y)$ is $n$ for generic non zero $Y_{\sigma}, \sigma=1, \ldots, 2 n$. For odd $n$, we set $A(Y)=\sum_{i=1}^{n} a_{i} \log F_{i}$, so that

Computing

$$
\nabla A(Y)=\left(a_{1} / F_{1}, \ldots, a_{n} / F_{n}, 0, \ldots, 0\right)^{T}
$$

$$
\left(\nabla A(Y)^{T} J(Y)\right)_{\sigma}= \begin{cases}\left(a^{T} G\right)_{\sigma} F_{k} & \text { if } \quad 1 \leq \sigma \leq n, \\ \left(a^{T} G\right)_{\sigma-n} B_{k} & \text { if } \quad n+1 \leq \sigma \leq 2 n,\end{cases}
$$

we then obtain $\nabla A(Y)^{T} J(Y)=0$. As $\nabla A(Y)$ is independent of the $\nabla c_{i}$ 's for generic $Y$, this means that $A(Y)$ is the last Casimir of the system.

Remark 3.3. If $n$ is even and the $\alpha_{i}$ 's non zero, then there exist $n$ real coefficients $a_{i}$ such that the system (2) is equivalent to the system

$$
Y^{\prime}=J(Y) \nabla H_{a}(Y)
$$

where $J(Y)$ is the matrix defined in $(6)$ and $H_{a}(Y)$ the Hamiltonian

$$
H_{a}(Y)=\sum_{i=1}^{n}\left(F_{i}-B_{i}+a_{i} \log F_{i}\right) \quad \text { for } \quad Y=(F, B) .
$$

As a matter of fact, we have

$$
\nabla H_{a}(Y)=\left(1+a_{1} / F_{1}, \ldots, 1+a_{n} / F_{n},-1, \ldots,-1\right)^{T}
$$


Thus the system (2) is equivalent to the system (11) if and only if we have $a^{T} G=-\alpha^{T}$ where $\alpha$ denotes the vector $\left(\alpha_{i}\right)_{i=1, \ldots, n}$ and $a$ the vector $\left(a_{i}\right)_{i=1, \ldots, n}$. Using the assumption that $G$ is invertible when $n$ is even, we get the result.

Given the form of the Casimir invariants, it now seems natural to consider the following change of variables:

$$
(F, B) \mapsto\left\{\begin{array}{rl}
c_{i} & =F_{i} B_{i}, \\
u_{i} & =\log \left(F_{i} / \sqrt{c_{i}}\right)
\end{array} \quad i=1, \ldots, n .\right.
$$

Under assumption (5), the transformation is a diffeomorphism and the inverse relations read

$$
F_{i}=\sqrt{c_{i}} \mathrm{e}^{u_{i}} \quad \text { and } \quad B_{i}=\sqrt{c_{i}} \mathrm{e}^{-u_{i}} .
$$

In the new coordinates, the Hamiltonian $H_{0}(Y)$ in $(10)$ writes now

$$
H_{0}(u, c)=2 \sum_{i=1}^{n} \sqrt{c_{i}} \sinh u_{i} .
$$

The classical Darboux-Lie theorem states that a Poisson system $y^{\prime}=A(y) \nabla H(y)$ can be locally written as a system of the form

where $J$ is the matrix

$$
z^{\prime}=J_{0} \nabla K(z) \quad \text { with } \quad J_{0}=\left(\begin{array}{cc}
J^{-1} & 0 \\
0 & 0
\end{array}\right),
$$

$$
J=\left(\begin{array}{cc}
0 & I \\
-I & 0
\end{array}\right),
$$

$I$ being the identity matrix of dimension $d$ and $2 d$ the rank of $A(y)$.

Here, for $F_{i}>0$ and $B_{i}>0, i=1, \ldots, n$, the matrix $J(Y)$ is of rank $n$ if $n$ is even and $n-1$ if $n$ is odd. We now show that using the change of unknowns (13), we can write a global Darboux-Lie transformation. We first state the following lemma:

Lemma 3.4. There exists an invertible matrix $M$ of order $n$ such that

$$
G=M A M^{T}
$$

where $A=J^{-1}$ if $n$ is even, and

$$
A=\left(\begin{array}{cc}
J^{-1} & 0 \\
0 & 0
\end{array}\right)
$$

where $J^{-1}$ is of dimension $n-1$ if $n$ is odd.

Proof. By the real Schur decomposition theorem (see for instance [4]), any real matrix $G$ can be brought to the form

$$
Q^{T} G Q=\left[\begin{array}{cccc}
R_{11} & R_{12} & \cdots & R_{1 m} \\
0 & R_{22} & \cdots & R_{2 m} \\
\vdots & \vdots & \cdots & \vdots \\
0 & 0 & \cdots & R_{m m}
\end{array}\right]
$$

where $R_{i i}$ is either a 1 -by- 1 real matrix or a 2 -by-2 real matrix with complex conjugate eigenvalues. Note that the matrix $Q$ involved in the transformation is real orthogonal, i.e. satisfies $Q^{T} Q=I$. Now, since $G$ is supposed to be skew-symmetric, we immediately get

$$
\begin{aligned}
& R_{i j}=0 \quad \text { for } j \neq i, \\
& R_{i i}^{T}=-R_{i i} .
\end{aligned}
$$


This means that $Q^{T} G Q$ is in fact a block-diagonal matrix with skew-symmetric blocks of dimension 1 or 2 on the diagonal, i.e. of the form $R_{i i}=0$ or

$$
R_{i i}=\left[\begin{array}{cc}
0 & \omega_{i} \\
-\omega_{i} & 0
\end{array}\right]
$$

Consider now the block diagonal matrix $D$ with diagonal blocks $D_{i i}=1$ if $R_{i i}=0$ and

$$
D_{i i}=\left[\begin{array}{cc}
\left|\omega_{i}\right|^{-1 / 2} & 0 \\
0 & \left|\omega_{i}\right|^{-1 / 2}
\end{array}\right]
$$

otherwise. The rescaled matrix $D^{T} Q^{T} G Q D$ is block-diagonal with diagonal blocks $D_{i i} R_{i i} D_{i i}$ either null or of the form

$$
\left[\begin{array}{cc}
0 & \pm 1 \\
\mp 1 & 0
\end{array}\right]
$$

It remains to notice that $D^{T} Q^{T} G Q D$ can be brought to the form stated in the lemma through a permutation matrix $P$ satisfying $P^{T} P=I\left(M\right.$ is then $\left.(Q D P)^{-T}\right)$.

Using this lemma, the following result can be easily proved:

Theorem 3.5. Suppose that $\alpha_{i}=0$ for $i=1, \ldots, n$ and that $G$ is skew symmetric of maximal rank. Let $M$ be the matrix of Lemma 3.4, and let $c$ and $u$ be the variables defined in (13). Then the transformation $(F, B) \mapsto(v, c)$, where $v=M^{-1} u$, defined a global Darboux-Lie change of variables from

$$
\left\{(F, B) \in \mathbb{R}^{2 n} \mid F_{i}>0 \quad \text { and } \quad B_{i}>0\right\} \quad \text { to } \quad\left\{(v, c) \in \mathbb{R}^{2 n} \mid c_{i}>0\right\} .
$$

In the coordinate system $z=(v, c)$, the system writes

$$
z^{\prime}=J_{0} \nabla K(z)
$$

where $J_{0}$ is of the form (14) with $J$ of dimension $n$ if $n$ is even and $n-1$ if $n$ is odd. The Hamiltonian $K(z)$ writes:

$$
K(z)=K(v, c)=\sum_{i=1}^{n} \sqrt{c_{i}} \sinh \left((M v)_{i}\right)=(\sqrt{c})^{T} \sinh (M v) .
$$

Note that if $n$ is odd, using the definition of $M$, the last component of $v$ is of the form $\sum_{i=1}^{n} a_{i} u_{i}$ where $a=\left(a_{i}\right)_{i=1}^{n}$ is in the kernel of $G$. Thus $v_{n}$ is the Casimir of Proposition 3.2.

\section{Change of UnKnowns}

In this section, we do not consider any longer neither that the $\alpha_{i}$ 's are necessarily zero, nor that $G$ is skewsymmetric. However, most of the invariants persist and the change of variable exhibited in (13) is still of interest. For a given $i$, a straightforward computation shows that

$$
\begin{aligned}
F_{i}^{\prime} B_{i} & =-\alpha_{i} F_{i} B_{i}+\sum_{j=1}^{n} G_{i j}\left(F_{j}+B_{j}\right) F_{i} B_{i} \quad \text { and } \\
B_{i}^{\prime} F_{i} & =\alpha_{i} B_{i} F_{i}-\sum_{j=1}^{n} G_{i j}\left(F_{j}+B_{j}\right) B_{i} F_{i} .
\end{aligned}
$$

This implies that $F_{i}^{\prime} B_{i}+F_{i} B_{i}^{\prime}=0$, i.e. that $c_{i}:=F_{i} B_{i}$ is constant along the trajectories of (2). Hence, equation (2) with $\alpha_{i} \neq 0, i=1, \ldots, n$, still possesses the $n$ invariants $c_{i}, i=1, \ldots, n$. The transformation 
considered in equations (13) is still relevant and will considerably simplify the analysis. Owing to the the simple relations $F_{i}+B_{i}=2 \sqrt{c_{i}} \cosh \left(u_{i}\right)$ and $u_{i}^{\prime}=F_{i}^{\prime} / F_{i}$, the system (2) can be written as

$$
\left\{\begin{aligned}
u_{i}^{\prime} & =-\alpha_{i}+2 \sum_{j=1}^{n} G_{i j} \sqrt{c_{j}} \cosh u_{j} \\
c_{i}^{\prime} & =0
\end{aligned} \text { for } \quad i=1, \ldots, n .\right.
$$

The boundary conditions are now

$$
u_{1}(0)=\log \left(P / \sqrt{c_{1}}\right) \quad \text { and } \quad u_{i}(0)=\frac{1}{2} \log R_{i}^{0} \quad \text { for } \quad i=2, \ldots, n,
$$

and

$$
u_{i}(L)=-\frac{1}{2} \log R_{i}^{L} \quad \text { for } \quad i=1, \ldots, 0 \quad \text { and } \quad u_{n}(L)=-\frac{1}{2} \log R_{\text {out }} .
$$

The original system (2-4) posed on the set $\left\{(F, B) \in \mathbb{R}^{2 n} \mid F_{i}>0 \quad\right.$ and $\left.\quad B_{i}>0\right\}$ thus reduces to equations (15$17)$ on the set $\left\{(u, c) \in \mathbb{R}^{2 n} \mid c_{i}>0\right\}$. It is worth mentioning that the boundary condition $u_{1}(0)=\log P / \sqrt{c_{1}}$ depends on $c_{1}$ while the others remain independent of the $c_{i}$ 's. As we see in further sections, this induces a different treatment according to the parity of $n$.

\section{Existence And Uniqueness Results FOR $n$ EVEn: A MODified PROBlem}

For even $n$, problem (15) with boundary conditions (16-17) is hardly tractable due to the term $\log \left(P / \sqrt{c_{1}}\right)$ in (16), for which it is hard to derive bounds (see next section). As a first step, we thus show the existence of a solution for the modified problem

$$
\left\{\begin{aligned}
u_{i}^{\prime} & =-\alpha_{i}+2 \sum_{j=1}^{n} G_{i j} \sqrt{c_{j}} \cosh u_{j} \\
c_{i}^{\prime} & =0
\end{aligned} \text { for } i=1, \ldots, n,\right.
$$

with the following boundary conditions

$$
u_{1}(0)=\frac{1}{2} \log R_{\text {in }} \quad \text { and } \quad u_{i}(0)=\frac{1}{2} \log R_{i}^{0} \quad \text { for } \quad i=2, \ldots, n,
$$

and

$$
u_{i}(L)=-\frac{1}{2} \log R_{i}^{L} \quad \text { for } \quad i=1, \ldots, n \quad \text { and } \quad u_{n}(L)=-\frac{1}{2} \log R_{\text {out }} .
$$

Here, $R_{\text {in }}$ is an unknown real number. With respect to the original variables, this corresponds to the boundary condition $F_{1}(0)=R_{\text {in }} B_{1}(0)$. For convenience, we denote in the sequel:

$$
R_{1}^{0}:=R_{\text {in }}, \quad R_{n}^{L}:=R_{\text {out }} \quad \text { and } \quad R^{0}=\left(R_{1}^{0}, \ldots, R_{n}^{0}\right)^{T}, \quad R^{L}=\left(R_{1}^{L}, \ldots, R_{n}^{L}\right)^{T} .
$$

In the ideal case where $G$ is skew-symmetric and $\alpha=0$, this problem is a Poisson system with boundary conditions. Using the same technique as in [3], we now show that it can be reformulated as a Cauchy problem for a system of integro-differential equations.

Integrating equations (18) from 0 to $L$, we find, for all $i=1, \ldots, n$ :

$$
u_{i}(L)-u_{i}(0)=-\alpha_{i} L+2 \sum_{j=1}^{n} G_{i j} \sqrt{c_{j}}\left\|\cosh u_{j}\right\|_{1}
$$


where $\left\|\cosh u_{j}\right\|_{1}$ is the $\mathrm{L}^{1}$ norm of $\cosh u_{i}$ in $[0, L]$. Hence, we see that

$$
\forall i=1, \ldots, n \quad 2 \sum_{j=1}^{n} G_{i j} \sqrt{c_{j}}\left\|\cosh u_{j}\right\|_{1}=-\frac{1}{2} \log R_{i}^{L}-\frac{1}{2} \log R_{i}^{0}+\alpha_{i} L .
$$

Defining successively $\mu=-\frac{1}{2} \log R^{L}-\frac{1}{2} \log R^{0}+L \alpha$ (where the log is applied component wise) and $q=G^{-1} \mu \in$ $\mathbb{R}^{n}$ (for non-singular $G$ ), equation (21) then reads

$$
\forall i=1, \ldots, n, \quad \sqrt{c_{i}}\left\|\cosh u_{i}\right\|_{1}=\frac{1}{2} q_{i} .
$$

This shows that the condition $q_{i}>0, i=1, \ldots, n$, is necessary to have the existence of a solution satisfying $c_{i}>0$ for all $i$. Moreover, under this condition, the system (18) is equivalent to the system

$$
\forall i=1, \ldots, n, \quad u_{i}^{\prime}=-\alpha_{i}+\sum_{j=1}^{n} G_{i j} q_{j} \frac{\cosh u_{j}}{\left\|\cosh u_{j}\right\|_{1}} .
$$

This equation is not an ordinary differential equation. It is defined for arbitrary, possibly negative, $q_{i}$ 's. The condition $q_{i}>0$ for all $i=1, \ldots, n$ thus appears as a condition for the problems (18-20) to possess a "physical" solution with non negative $c_{i}$ 's.

Note that if $u=\left(u_{i}\right)_{i=1}^{n}$ satisfies (23) with the boundary conditions (19), then by definition of $q$, the boundary conditions $(20)$ are also satisfied.

We begin by showing an existence result for a general problem of the form (23) with Cauchy boundary conditions at $x=0$ (see [3]):

Proposition 5.1. Let $\beta \in \mathbb{R}^{n}$, A be a matrix of size $n$, and $v^{0} \in \mathbb{R}^{n}$. There exists a vector $v$ with smooth coefficients $v_{i}(x), i=1, \ldots, n$ defined on $[0, L]$, solution of the equations:

$$
\text { For } i=1, \ldots, n\left\{\begin{array}{l}
v_{i}^{\prime}(x)=\beta_{i}+\sum_{j=1}^{n} A_{i j} \frac{\cosh v_{j}(x)}{\left\|\cosh v_{j}\right\|_{1}}, \text { for } x \in[0, L], \\
v_{i}(0)=v_{i}^{0} .
\end{array}\right.
$$

Using this result with $\beta_{i}=-\alpha_{i}, v_{i}^{0}=\frac{1}{2} \log R_{i}^{0}$ for $i=1, \ldots, n$ and $A_{i j}=G_{i j} q_{j}$ for $i, j=1, \ldots, n$, we deduce immediately the following theorem:

Theorem 5.2. Suppose that $n$ is even and let

$$
\mu=-\frac{1}{2} \log R^{L}-\frac{1}{2} \log R^{0}+L \alpha .
$$

The system (18) together with the boundary conditions (19) and (20) possesses a smooth solution with $c_{i}>0$ for $i=1, \ldots, n$, if and only if the components of $q=G^{-1} \mu$ satisfy

$$
q_{i}>0 \quad \text { for } \quad i=1, \ldots, n \text {. }
$$

In this case, one has the relation

$$
c_{i}=\left(\frac{q_{i}}{2\left\|\cosh u_{i}\right\|_{1}}\right)^{2} \quad \text { for } \quad i=1, \ldots, n .
$$


Later on, we will discuss condition (26) and show that it is satisfied in situations of practical interest (see Prop. 6.3 below).

Proof. (of Prop. 5.1) The function $v$ is a solution of (24) if and only if it is a solution of the fixed-point problem

$$
v=\Phi(v)
$$

where $\Phi$ is defined by

$$
\Phi(v)_{i}(x)=v_{i}^{0}+\beta_{i} x+\sum_{j=1}^{n} A_{i j} \int_{0}^{x} \frac{\cosh v_{j}(t)}{\left\|\cosh v_{j}\right\|_{1}} \mathrm{~d} t, \quad \text { for } \quad i=1, \ldots, n .
$$

On the space $\left(\mathrm{L}^{\infty}\right)^{n}$, we define the norm $\|v\|_{\infty}=\max _{i=1}^{n}\left\|v_{i}\right\|_{\mathrm{L}^{\infty}}$. If $v \in\left(\mathrm{L}^{\infty}\right)^{n}$, we have for all $x \in[0, L]$,

$$
\|\Phi(v)\|_{\infty} \leq\left\|v^{0}\right\|_{\infty}+L\|\beta\|_{\infty}+\|A\|_{\infty},
$$

where $\|\cdot\|_{\infty}$ denotes either the norm on $\left(\mathrm{L}^{\infty}\right)^{n}$ or the standard infinity norm for vectors and matrices in $\mathbb{R}^{n}$. Note that we used the fact that

$$
0 \leq \int_{0}^{x} \frac{\cosh v_{j}}{\left\|\cosh v_{j}\right\|_{1}} \leq 1
$$

Moreover, as $\left\|\cosh v_{j}\right\|_{1} \geq L$ for $j=1, \ldots, n$, we also have for all $v \in\left(\mathrm{L}^{\infty}\right)^{n}$,

$$
\forall i=1, \ldots, n, \quad\left|\frac{\mathrm{d} \Phi(v)_{i}(x)}{\mathrm{d} x}\right| \leq\left|\beta_{i}\right|+\frac{1}{L} \sum_{j=1}^{n}\left|A_{i j}\right| \cosh \left\|v_{j}\right\|_{\mathrm{L}^{\infty}}
$$

and hence

$$
\left\|\Phi(v)^{\prime}\right\|_{\infty} \leq\|\beta\|_{\infty}+\frac{1}{L}\|A\|_{\infty} \cosh \|v\|_{\infty} .
$$

Thus $\Phi$ is a map from $\left(\mathrm{L}^{\infty}\right)^{n}$ to $\left(\mathrm{W}^{1, \infty}\right)^{n}$. Now, for $u$ and $v$ in $\left(\mathrm{L}^{\infty}\right)^{n}$ and for all $i=1, \ldots, n$, we have:

$$
\begin{aligned}
\Phi(u)_{i}(x)-\Phi(v)_{i}(x)=\sum_{j=1}^{n} A_{i j} & \frac{1}{\left\|\cosh u_{j}\right\|_{1}} \int_{0}^{x}\left(\cosh u_{j}(t)-\cosh v_{j}(t)\right) \mathrm{d} t \\
& +\sum_{j=1}^{n} A_{i j}\left(\int_{0}^{x} \frac{\cosh v_{j}(s)}{\left\|\cosh v_{j}\right\|_{1}} \mathrm{~d} s\right) \frac{1}{\left\|\cosh u_{j}\right\|_{1}} \int_{0}^{L}\left(\cosh v_{j}(t)-\cosh u_{j}(t)\right) \mathrm{d} t .
\end{aligned}
$$

Using (29) and the fact that the $\mathrm{L}^{1}$ norms of $\cosh u_{j}$ and $\cosh v_{j}$ are greater than $L$, we get the bound

$$
\left|\Phi(u)_{i}(x)-\Phi(v)_{i}(x)\right| \leq 2 \sum_{j=1}^{n}\left|A_{i j}\right||| \cosh u_{j}-\cosh v_{j} \|_{L^{\infty}},
$$

for all $i=1, \ldots, n$. Hence, if $u$ and $v$ satisfy $\|u\|_{\infty} \leq M$ and $\|v\|_{\infty} \leq M$, we have

$$
\|\Phi(u)-\Phi(v)\|_{\infty} \leq 2\|A\|_{\infty}(\sinh M)\|u-v\|_{\infty} .
$$

In a similar way, we find

$$
\left\|\Phi(u)^{\prime}-\Phi(v)^{\prime}\right\|_{\infty} \leq \frac{1}{L}\left(1+\frac{\cosh M}{L}\right)\|A\|_{\infty}(\sinh M)\|u-v\|_{\infty} .
$$


This shows that $\Phi$ is continuous from $\left(\mathrm{L}^{\infty}\right)^{n}$ to $\left(\mathrm{W}^{1, \infty}\right)^{n}$. As $[0, L]$ is bounded, the embedding $\mathrm{W}^{1, \infty} \rightarrow C(0, L)$ is compact, so that $\Phi$ defines a continuous compact application

$$
\Phi:\left(\mathrm{L}^{\infty}\right)^{n} \longrightarrow\left(\mathrm{L}^{\infty}\right)^{n}
$$

with bounded range $K \subset C(0, L)^{n}$ (see (28)). Due to Schauder's theorem, we can assert that $\Phi$ has a fixed point $v$ in $K: v$ is a continuous solution of (24). An easy induction then shows that $v \in C^{\infty}(0, L)$ and this concludes the proof.

Remark 5.3. In the case where $\beta_{i}=0$, the system (24) is invariant by scaling of the interval $[0, L]$. Indeed, we see that if the functions $v_{i}, i=1, \ldots, n$, are solutions of $(24)$ on $[0, L]$, then the functions $y \mapsto v_{i}(L y)$ are solutions of the same equation on $(0,1)$ (with the $\mathrm{L}^{1}$ norm on $(0,1)$ ). This is easily seen by change of variables.

In [3] we proved that for $n=2, \alpha_{1}=\alpha_{2}=0$ and $G$ skew-symmetric, the solution given in Theorem 5.2 is unique. As we will see now, uniqueness for higher dimensions is only proved here under a smallness hypothesis on the data. As before, we first state a general result for problems of the form (24):

Proposition 5.4. There exists a number $\varepsilon>0$ such that for every matrix $A$ and all vectors $v^{0}$ and $\beta$ satisfying

$$
\left\|v^{0}\right\|_{\infty}+L\|\beta\|_{\infty}+\|A\|_{\infty}<\varepsilon
$$

the solution $v$ of Proposition 5.1 is unique. Moreover, the sequence $v^{(k)}$ defined for $k \geq 0$ by $v^{(k+1)}=\Phi\left(v^{(k)}\right)$ where $\Phi$ is defined by $(27)$ and $v^{(0)}=0$ converges toward $v$.

The multiplication by $L$ in inequality (32) owes to homogeneity considerations (see (28)). Before proving this result, we show how it yields a uniqueness result for problem (18-20). Recall that for given positive numbers $R_{i}^{0}, R_{i}^{L}$ and $\alpha_{i}$ we set $\mu=-\frac{1}{2} \log R^{L}-\frac{1}{2} \log R^{0}+L \alpha$ and $q=G^{-1} \mu$. For $\delta>0$, we define the set

$$
\Omega_{\delta}=\left\{R_{i}^{0}, R_{i}^{L}, \alpha_{i} \in \mathbb{R}^{3 n} \quad|\quad| R_{i}^{0}-1|<\delta, \quad| R_{i}^{L}-1 \mid<\delta \quad \text { and } \quad\left|\alpha_{i}\right|<\delta\right\} .
$$

Note that for all $\delta>0, \Omega_{\delta}$ is a domain of $\mathbb{R}^{3 n}$ containing the point $(1,1,0)$ (i.e. $R_{i}^{1}=1, R_{i}^{L}=1$ and $\alpha_{i}=0$ for all $i=1, \ldots, n)$. Using Proposition 5.4 with $\beta=-\alpha, v^{0}=\frac{1}{2} \log R^{0}$ and $A=G \cdot \operatorname{diag}(q)$, we obtain the following result:

Theorem 5.5. There exists a real number $\delta$ such that if $\left(R^{0}, R^{L}, \alpha\right) \in \Omega_{\delta}$ satisfies

$$
\forall i=1, \ldots, n, \quad q_{i}>0
$$

with $\mu=-\frac{1}{2} \log R^{L}-\frac{1}{2} \log R^{0}+L \alpha$ and $q=G^{-1} \mu$, then the solution $u^{*}$ of the equations (18-20) is unique. Moreover, the sequence of functions $u^{(k)}$ defined on $[0, L]$ by

$$
\begin{aligned}
u_{i}^{(0)} & =0, \quad i=1, \ldots, n, \\
\left(u_{i}^{(k+1)}\right)^{\prime} & =-\alpha_{i}+\sum_{j=1}^{n} G_{i j} q_{j} \frac{\cosh u_{j}^{(k)}}{\left\|\cosh u_{j}^{(k)}\right\|_{1}}, \quad u_{i}^{(k+1)}(0)=\frac{1}{2} \log R_{i}^{0}, \quad i=1, \ldots, n,
\end{aligned}
$$

converges towards $u^{*}$.

Proof. (of Prop. 5.4) Under assumption (32), we can see by using (28) that for all $v \in\left(\mathrm{L}^{\infty}\right)^{n}$, $\Phi(v)$ takes its values in the ball $B_{\infty}(0, \varepsilon)$ of $\left(\mathrm{L}^{\infty}\right)^{n}$. As a consequence, if $v$ and $u$ are two solutions of $(24)$, they satisfy $\|v\|_{\infty}<\varepsilon$ and $\|u\|_{\infty}<\varepsilon$.

Using equation (31) and the bound $\|A\|_{\infty}<\varepsilon$, it follows that

$$
\|\Phi(u)-\Phi(v)\|_{\infty} \leq 2 \varepsilon(\sinh \varepsilon)\|u-v\|_{\infty} .
$$


Let $k=2 \varepsilon(\sinh \varepsilon)$. For sufficiently small $\varepsilon, k<1$ so that $\Phi$ is a contraction from $B_{\infty}(0, \varepsilon)$ to itself. Hence, $u=v=u^{*}$ and the sequence $u^{(k)}$ converges toward $u^{*}$.

Remark 5.6. In [3], the following slightly different result is shown: for $\beta=0$ and a given $v^{0}$, there exists $\varepsilon$ such that whenever $\|A\|_{\infty}<\varepsilon$, the solution is unique. Here, as we wish to solve the original problem with a given pump power $P$, we need to derive estimates that hold uniformly with respect to the initial value $v^{0}$.

\section{EXISTENCE AND UNIQUENESS RESUlts FOR $n$ EVEN: THE INITIAL EQUATIONS}

Comparing equations (15-17) and (18-20) shows that solving the first system is equivalent to finding a value of $R_{\text {in }}$ satisfying $\sqrt{R_{\text {in }}}=P / \sqrt{c_{1}}$, where $c_{1}$ depends on $R_{\text {in }}$, for fixed $\alpha_{i}, R_{i}^{L}(i=1, \ldots, n), R_{i}^{0}(i=2, \ldots, n)$ and $P$. Using relation (22), this reads

$$
R_{\text {in }}=\frac{4 P^{2}}{q_{1}^{2}}\left\|\cosh u_{1}\right\|_{1}^{2}=: g\left(R_{\text {in }}\right) .
$$

Note that in order to define the function $g$, the data have to be taken in the set $\Omega_{\delta}$ with $\delta$ sufficiently small.

Here, we show that under conditions on $P$ and the data, we can find a unique solution of this equation if the data are "small": this means in particular that the solution $R_{\text {in }}=g\left(R_{\text {in }}\right)$ is close to 1 , or, equivalently, that $\sqrt{c_{1}}$ is close to $P$ or $\frac{1}{2} q_{1}\left\|\cosh u_{1}\right\|_{1}^{-1}$ is close to $P$.

For $\delta>0$, we introduce the following set:

$$
U_{\delta}:=\left\{\quad\left(R_{j}^{0}\right)_{j=2}^{n}, \quad\left(R_{i}^{L}, \alpha_{i}\right)_{i=1}^{n} \quad|\quad| R_{j}^{0}-1|<1, \quad| R_{i}^{0}-1 \mid<1, \quad \text { and } \quad\left|\alpha_{i}\right|<\delta\right\} .
$$

We set $X=\left(R_{2}^{0}, \ldots, R_{n}^{0}, R_{1}^{L}, \ldots, R_{n}^{L}, \alpha_{1}, \ldots, \alpha_{n}\right) \in \mathbb{R}^{3 n-1}$ an element of $U_{\delta}$. Note that if $\left|R_{1}^{0}-1\right|<\delta$ then $\left(R_{1}^{0}, X\right) \in \Omega_{\delta}$.

We write $q_{j}(R, X)$ for the coefficients of $q=G^{-1} \mu$ where $\mu$ depends on $(R, X)$ via the equation (25) for $R_{1}^{0}=R_{\text {in }}$.

We first note the following: suppose that $X \in U_{\delta}$ is fixed, then as $G_{11}=0$, for all $R_{1}^{0}, q_{1}$ depends only on $X$, and is written $q_{1}(X)$.

Now for fixed $X \in U_{\delta}$, assuming that $P$ is close to $\sqrt{c_{1}}$ means that $P$ is close to $\frac{1}{2} q_{1}(X)\left\|\cosh u_{1}\right\|_{1}^{-1}$. If $\delta$ is sufficiently small, the solution $u$ is close to zero, so that $\left\|\cosh u_{1}\right\|_{1}$ is approximatively equal to $L$. Hence it appears necessary to consider values of $P$ in a neighborhood of $\frac{q_{1}(X)}{2 L}$. A condition of this type indeed ensures existence and uniqueness of the solution:

Theorem 6.1. There exists a real number $\delta>0$, such that if $X \in U_{\delta}$ satisfies:

$$
q_{1}(X)>0 \quad \text { and } \quad \forall R \in I_{\delta}:=[1,1+\delta], \quad q_{j}(R, X) \geq 0 \quad \text { for } \quad j=2, \ldots, n,
$$

and if $P$ is a real number such that

$$
\frac{q_{1}(X)}{2 L} \leq P \leq \frac{q_{1}(X)}{2 L}\left(1+\frac{\delta}{4}\right)
$$

then there exists a unique $R_{\mathrm{in}} \in[1,1+\delta]$ such that the solution $(u, c)$ of the equations $(18-20)$ for the data $R_{\text {in }}$ and $X$ satisfies

$$
R_{\text {in }}=\frac{P^{2}}{c_{1}}=\frac{4 P^{2}}{q_{1}^{2}(X)}\left\|\cosh u_{1}\right\|_{1}^{2} .
$$

Hence $(u, c)$ is the unique solution of the initial problem $(15-17)$. If we have in addition $q_{j}\left(R_{\text {in }}, X\right)>0$ for $j=2, \ldots, n$, then $(F, B)$ defined by $F_{i}=\sqrt{c_{i}} \mathrm{e}^{u_{i}}$ and $B_{i}=\sqrt{c_{i}} \mathrm{e}^{-u_{i}}$ for $i=1, \ldots, n$ is the unique positive solution of $(2-4)$. 
Proof. Let $X \in U_{\delta}$ and $R \in I_{\delta}:=[1,1+\delta]$. We recall that for $\delta$ sufficiently small, the function $\Phi$ defined in (27) satisfies the following estimate: there exist continuous functions $M(\delta)>0$ and $\rho(\delta)>0$ such that $M(\delta) \rightarrow 0$, $\rho(\delta) \rightarrow 0$ as $\delta \rightarrow 0$, and such that

$$
\forall u, v \in B_{\infty}(0, M(\delta)), \quad\|\Phi(u)-\Phi(v)\|_{\infty} \leq \rho(\delta)\|u-v\|_{\infty}
$$

In the following, when $X$ is fixed, we write $\Phi(u, R)$ instead of $\Phi(u)$ to fix the value of $R=R_{1}^{0}$.

Lemma 6.2. There exists $\delta_{0}>0$ such that for $\delta<\delta_{0}$ and for $X \in U_{\delta}, R \in I_{\delta}, \tilde{R} \in I_{\delta}$, and $u$ and $\tilde{u}$ solutions of

$$
u=\Phi(u, R) \quad \text { and } \quad \tilde{u}=\Phi(\tilde{u}, R),
$$

then we have

where $C(G)$ depends only on $G$.

$$
\|u-\tilde{u}\|_{\infty} \leq C(G)|R-\tilde{R}|
$$

We postpone the proof of this lemma. Let $\delta<\delta_{0}$, and let $X \in U_{\delta}$ satisfy (38). For $R \in I_{\delta}$, we define the function

$$
g(R)=\frac{4 P^{2}}{q_{1}(X)^{2}}\left\|\cosh u_{1}\right\|_{1}^{2}
$$

where $u$ is solution of $u=\Phi(u, R)$. Suppose that $P$ satisfies (39). Then we have for $R \in I_{\delta}$,

$$
g(R) \geq \frac{4 L^{2} P^{2}}{q_{1}(X)^{2}} \geq 1 .
$$

Moreover, as $X$ satisfies (38), $\alpha_{1} \geq 0$, and the coefficients $G_{1 j}$ are non-positive for $j=2, \ldots, n$, so that $u_{1}^{\prime}(x) \leq 0$ for $x \in[0, L]$ (see (23)). It follows that $u_{1}(x) \leq u_{1}(0)=\log \sqrt{R}$ and

$$
g(R) \leq \frac{4 L^{2} P^{2}}{q_{1}(X)^{2}}\left(\cosh u_{1}(0)\right)^{2} .
$$

Using

For $R \in[1,1+\delta]$ we get

$$
\cosh u_{1}(0)=\frac{R+1}{2 \sqrt{R}}
$$

Under condition (39), this inequality becomes

$$
g(R) \leq \frac{L^{2} P^{2}(2+\delta)^{2}}{q_{1}(X)^{2}(1+\delta)}
$$

$$
g(R) \leq \frac{(2+\delta)^{2}}{4(1+\delta)}\left(1+\frac{\delta}{4}\right)^{2}
$$

Eventually, since for sufficiently small $\delta_{0}$ and $\delta_{0}>\delta>0$

$$
(2+\delta)^{2}\left(1+\frac{\delta}{4}\right)^{2} \leq 4(1+\delta)^{2}
$$

this simplifies into

$$
g(R) \leq 1+\delta
$$

As a consequence, and provided once again that condition (39) is satisfied, $g$ maps $I_{\delta}$ to itself. 
Now considering $u$ and $\tilde{u}$ the respective solutions of $u=\Phi(u, R)$ and $\tilde{u}=\Phi(\tilde{u}, \tilde{R})$, and taking $R$ and $\tilde{R}$ in $I_{\delta}$, we compute

$$
g(R)-g(\tilde{R})=\frac{4 P^{2}}{q_{1}(X)^{2}}\left(\left\|\cosh u_{1}\right\|_{1}^{2}-\left\|\cosh \tilde{u}_{1}\right\|_{1}^{2}\right) .
$$

From $\|u\|_{\infty} \leq M(\delta)$ and $\|\tilde{u}\|_{\infty} \leq M(\delta)$, we then have

$$
|g(R)-g(\tilde{R})| \leq \frac{4 L^{2} P^{2}}{q_{1}(X)^{2}} 2(\cosh M(\delta))(\sinh M(\delta))\|u-\tilde{u}\|_{\infty}
$$

and upon using the bound (26) and Lemma 6.2, we recover the following expression

$$
|g(R)-g(\tilde{R})| \leq 2 C(G)\left(1+\frac{\delta}{4}\right)^{2}(\cosh M(\delta))(\sinh M(\delta))|R-\tilde{R}| .
$$

Since $M(\delta)$ tends to 0 when $\delta \rightarrow 0$, we may assume that $\delta_{0}$ is small enough for $g$ to be a contraction from $I_{\delta}$ to itself provided $\delta<\delta_{0}$. This proves the uniqueness of the fixed-point of $g$.

Proof. (of Lem. 6.2) Let $\delta_{0}$ be such that $\rho(\delta) \leq \frac{1}{2}$ for $\delta \leq \delta_{0}$. As $u$ and $\tilde{u}$ are in $B_{\infty}(0, M(\delta)$ ), we get straightforwardly

$$
\begin{aligned}
\|u-\tilde{u}\|_{\infty} & =\|\Phi(u, R)-\Phi(\tilde{u}, \tilde{R})\|_{\infty} \\
& \leq\|\Phi(u, R)-\Phi(\tilde{u}, R)\|_{\infty}+\|\Phi(\tilde{u}, R)-\Phi(\tilde{u}, \tilde{R})\|_{\infty} \\
& \leq \frac{1}{2}\|u-\tilde{u}\|_{\infty}+\|\Phi(\tilde{u}, R)-\Phi(\tilde{u}, \tilde{R})\|_{\infty} .
\end{aligned}
$$

Using the expression of $\Phi$, it then follows that

$$
\|u-\tilde{u}\|_{\infty} \leq|\log R-\log \tilde{R}|+2 \sum_{j=2}^{n}\left|q_{j}-\tilde{q}_{j}\right|\left|G_{i j}\right|,
$$

where $q_{j}$ and $\tilde{q}_{j}$ denote the components of $q$ as functions respectively of $R$ and $\tilde{R}$ and for fixed $X$ (recall that $q_{1}(X)$ does not depend on $R$ ). As $q=G^{-1} \mu$ with $\mu$ defined in (25), we see that there exits $C(G)$ depending only on $G$ such that

$$
\|u-\tilde{u}\|_{\infty} \leq C(G)|\log R-\log \tilde{R}| .
$$

Owing to the fact that both $R$ and $\tilde{R}$ belong to $I_{\delta}$, the inequality $|\log R-\log \tilde{R}| \leq|R-\tilde{R}|$ leads to the result.

We conclude this section by showing that conditions (26) and (38) are fulfilled in the situation where $G$ has non zero coefficient only on the upper and lower first diagonals, where the $\alpha_{i}$ 's are zero, and where most of the reflectivity coefficients $R_{i}^{0}$ and $R_{i}^{L}$ are equal to 1 .

Proposition 6.3. Suppose that $G$ satisfies

$$
G_{i j}=0 \quad \text { for } \quad|i-j|<1 .
$$

Suppose moreover that for all $i=1, \ldots, n, \alpha_{i}=0$,

$$
\forall i=2, \ldots, n, \quad R_{i}^{0}=1 \quad \text { and } \quad \forall i=1, \ldots, n-1, \quad R_{i}^{L}=1 .
$$

For all $R_{\mathrm{out}}=: R_{n}^{L}<1$ and $R_{\mathrm{in}}>1$, let $\mu$ be defined as in (25) and $q=G^{-1} \mu$. Then we have $q_{j}>0$ for all $j=1, \ldots, n$. In particular, condition (38) is satisfied. 
Proof. Under the hypotheses of the proposition, we have $\mu_{i}=0$ for $i=2, \ldots, n-1, \mu_{1}=-\frac{1}{2} \log R_{\text {in }}$ and $\mu_{n}=-\frac{1}{2} \log R_{\text {out }}$. Due to (40) the components of $q=G^{-1} \mu$ may then be written as

$$
q_{i}=\left\{\begin{array}{llll}
\nu_{i}\left(\frac{1}{2} \log R_{\text {in }}\right) & \text { for } & i & \text { even }, \\
\nu_{i}\left(-\frac{1}{2} \log R_{\text {out }}\right) & \text { for } & i & \text { odd },
\end{array}\right.
$$

where $\nu_{i}$ are positive numbers and the result follows at once.

\section{Existence AND UNIQUENESS RESUlts FOR $n$ ODD}

In this section, we prove existence and uniqueness of the solution of (15) with initial conditions (16-17) under the assumption that the data are small enough. Note that for boundary conditions of the form (19-20), the system for odd $n$ cannot be directly written in the form (23) since the matrix $G$ is not invertible.

Let $n=2 p+1$ and assume that $G$ is of rank $2 p$. Then there exist vectors $a$ and $b$ such that $a^{T} G=0$ and $G b=0$ (if $G$ is skew symmetric, we can take $a=b$ ). For convenience and with no loss of generality, we may assume that the first coefficients $a_{1}$ and $b_{1}$ of $a$ and $b$ are non zero. Similarly to the proof of Proposition 3.2, we have

$$
\frac{\mathrm{d}}{\mathrm{d} x}\left(\sum_{i=1}^{n} a_{i} \log F_{i}\right)=-\sum_{i=1}^{n} a_{i} \alpha_{i}
$$

and upon integrating from 0 to $L$

$$
\sum_{i=1}^{n} a_{i}\left(\log F_{i}(L)-\log F_{i}(0)\right)=-\sum_{i=1}^{n} a_{i} \alpha_{i} L .
$$

Applying the change of coordinates (see (13))

$$
\log F_{i}(L)-\log F_{i}(0)=u_{i}(L)-u_{i}(0),
$$

and taking into account the boundary conditions (16) and (17) leads to

$$
\sum_{i=1}^{n} a_{i}\left(u_{i}(L)-u_{i}(0)+\alpha_{i} L\right)=0
$$

Since $a_{1} \neq 0$, we then get

$$
u_{1}(0)=u_{1}(L)+\alpha_{1} L+\sum_{i=2}^{n} \frac{a_{i}}{a_{1}}\left(u_{i}(L)-u_{i}(0)+\alpha_{i} L\right)
$$

and inserting $u_{1}(0)=\log P-\log \sqrt{c_{1}}$, we find that

$$
\log \sqrt{c_{1}}=\log P+\frac{1}{2} \log R_{1}^{L}-\alpha_{1} L+\sum_{i=2}^{n} \frac{a_{i}}{a_{1}}\left(\frac{1}{2} \log \left(R_{i}^{L} R_{i}^{0}\right)-\alpha_{i} L\right)
$$

with the notation $R_{n}^{L}=R_{\text {out }}$.

This shows that for odd $n$, the constant $c_{1}$ depends explicitly on the parameters of the problem: the boundary condition in $u_{1}(0)$ may be put in the form $u_{1}(0)=\log \sqrt{R_{1}^{0}}$ where $R_{1}^{0}=P^{2} / c_{1}$ is given. 
Consider now the system (15) together with boundary conditions (16) and (17). Integrating from 0 to $L$, we get for $i=1, \ldots, n$,

$$
u_{i}(L)-u_{i}(0)+\alpha_{i} L=2 \sum_{j=1}^{n} G_{i j} \sqrt{c_{j}}\left\|\cosh u_{j}\right\|_{1} .
$$

Denoting respectively $\mu$ and $q$ the vectors with components respectively $\mu_{i}=u_{i}(L)-u_{i}(0)+\alpha_{i} L$ and $q_{i}=$ $2 \sqrt{c_{i}}\left\|\cosh u_{i}\right\|_{1}$, the system reads $G q=\mu$. Now, since $G$ is of rank $2 p=n-1$, the dimension of Null $G^{T}$ is 1 and we have

$$
\mathbb{R}^{n}=\operatorname{Null} G^{T} \oplus G\left(\mathbb{R}^{n}\right)
$$

with Null $G^{T}=\operatorname{Span}(a)=\left(G\left(\mathbb{R}^{n}\right)\right)^{\perp}$. Now by definition of $c_{1}, \mu^{T} a=0$, so that $\mu \in G\left(\mathbb{R}^{n}\right)$. This means that $q$ is determined up to an element of the kernel of $G$ : if $q^{0}$ is a particular solution of the system $G q=\mu$, the general solution $q$ can thus be written as

$$
q=q^{0}+\lambda b
$$

where $\lambda \in \mathbb{R}$. Now writing the first component of this equation yields

$$
2 \sqrt{c_{1}}\left\|\cosh u_{1}\right\|_{1}=q_{1}^{0}+\lambda b_{1},
$$

and we then get

$$
\lambda=\frac{1}{b_{1}}\left(2 \sqrt{c_{1}}\left\|\cosh u_{1}\right\|_{1}-q_{1}^{0}\right)=\frac{1}{b_{1}}\left(2 \frac{P}{\sqrt{R_{\text {in }}}}\left\|\cosh u_{1}\right\|_{1}-q_{1}^{0}\right)
$$

where $R_{\text {in }}=R_{1}^{0}$ is fixed. We sum up these results in the following proposition.

Proposition 7.1. Let $n=2 p+1$ and assume that $G$ is of rank $2 p$. Consider the vectors $a=\left(a_{i}\right)_{i=1}^{n}$ and $b=\left(b_{i}\right)_{i=1}^{n}$ such that $a^{T} G=0$ and $G b=0$ and assume that $a_{1}=b_{1}=1$. Then the system (15-17) is equivalent to the following system in $u$ and $\lambda$ :

$$
\begin{aligned}
u_{i}^{\prime}(x) & =-\alpha_{i}+\sum_{j=1}^{n} G_{i j}\left(q_{j}^{0}+\lambda b_{j}\right) \frac{\cosh u_{j}(x)}{\left\|\cosh u_{j}\right\|_{1}} & & \text { for } i=1, \ldots, n \text { and } x \in[0, L], \\
\lambda & =\frac{2 P}{\sqrt{R_{1}^{0}}\left\|\cosh u_{1}\right\|_{1}-q_{1}^{0},} & & \text { for } i=1, \ldots, n .
\end{aligned}
$$

Here

$$
R_{1}^{0}=\mathrm{e}^{\left(2 L \sum_{i=1}^{n} a_{i} \alpha_{i}\right)}\left(R_{1}^{L}\right)^{-1} \prod_{i=2}^{n}\left(R_{i}^{L} R_{i}^{0}\right)^{-a_{i}}
$$

depends only on the data of the problem, and $q^{0}$ is a given particular solution of $G q=\mu$ with $\mu$ defined by

$$
\mu_{i}=-\frac{1}{2} \log R_{i}^{L}-\frac{1}{2} \log R_{i}^{0}+\alpha_{i} L .
$$

Using the notations of previous section, $X$ denotes an element of $U_{\delta}$ defined in (37). As $R_{1}^{0}$ is given by (45), we see that the vector $q_{i}^{0}$ depends only on $X$, and we write $q_{i}^{0}(X)$. Note that if $X \in U_{\delta}$ with $\delta$ sufficiently small, $R_{1}^{0}$ is close to 1 and $q^{0}$ is closed to 0 . Using this fact leads to the following result:

Theorem 7.2. There exists a real number $\delta_{0}$ such that for all $\delta<\delta_{0}$ the following proposition holds true: if $X \in U_{\delta}$ is such that

$$
\forall \lambda \in[0, \delta], \quad q_{i}^{0}(X)+\lambda b_{i}>0 \quad \text { for } \quad i=1, \ldots, n,
$$


then for any real number $P$ satisfying

$$
\frac{q_{1}^{0}(X) \sqrt{R_{1}^{0}}}{2 L} \leq P \leq\left(q_{1}^{0}(X)+\delta\right) \frac{R_{1}^{0}}{L\left(R_{1}^{0}+1\right)},
$$

where $R_{1}^{0}$ is defined by (45), problem (44) has a unique solution $(u, \lambda)$. Moreover, $\lambda \in[0, \delta]$ and $u$ is the unique solution of the system (15-17) with

$$
\sqrt{c_{i}}=\frac{q_{i}^{0}+\lambda b_{i}}{2\left\|\cosh u_{i}\right\|_{1}} \quad \text { for } \quad i=2, \ldots, n
$$

and $c_{1}$ given by (43).

Proof. For any $\lambda \in \mathbb{R}$ and $X \in U_{\delta}$, we define the application $\Phi_{\lambda}$ as

$$
\Phi_{\lambda}(v)_{i}(x)=\frac{1}{2} \log R_{i}^{0}-\alpha_{i} x+\sum_{j=1}^{n} G_{i j}\left(q_{i}^{0}(X)+\lambda b_{i}\right) \int_{0}^{x} \frac{\cosh v_{j}(t)}{\left\|\cosh v_{j}\right\|_{1}} \mathrm{~d} t, \quad \text { for } \quad i=1, \ldots, n .
$$

Before going on, we first state without proof the following result, which is a direct consequence of the bounds (28) and (31).

Lemma 7.3. There exist $\delta_{0}>0$ and continuous functions $M(\delta)>0$ and $\rho(\delta)>0$ satisfying $M(\delta) \rightarrow 0$ and $\rho(\delta) \rightarrow 0$ as $\delta \rightarrow 0$, such that for all $\delta<\delta_{0}$, for all $X \in U_{\delta}$ and for all $\lambda \in[0, \delta], \Phi_{\lambda}$ satisfies

$$
\forall u, v \in B_{\infty}(0, M(\delta)) \subset\left(\mathrm{L}^{\infty}\right)^{n}, \quad\left\|\Phi_{\lambda}(u)-\Phi_{\lambda}(v)\right\|_{\infty} \leq \rho(\delta)\|u-v\|_{\infty} .
$$

Now, if $\delta<\delta_{0}$ and $X \in U_{\delta}$, then for all $\lambda \in[0, \delta]$, there exists a unique $u^{(\lambda)}$ solution of $u^{(\lambda)}=\Phi_{\lambda}\left(u^{(\lambda)}\right)$. Thus, we can define the function

$$
g(\lambda)=\frac{2}{\sqrt{R_{1}^{0}}} P\left\|\cosh u_{1}^{(\lambda)}\right\|_{1}-q_{1}^{0}(X) .
$$

It remains to show that $g$ has a unique fixed point in $[0, \delta]$. Proceeding as in the proof of Theorem 6.1 , it can be seen that condition (46) implies that $g$ maps $[0, \delta]$ into itself. Moreover, we see that for $\lambda$ and $\tilde{\lambda}$ in $[0, \delta]$ we have

$$
|g(\lambda)-g(\tilde{\lambda})| \leq \frac{2}{\sqrt{R_{1}^{0}}} P(\sinh M(\delta))\left\|u^{(\lambda)}-u^{(\tilde{\lambda})}\right\|_{\infty} .
$$

We may now conclude, as in Lemma 6.2 , that for $\delta_{0}$ sufficiently small, the function $g$ is a contraction from $[0, \delta]$ to itself. This finishes the proof.

\section{NumERICAL EXPERIMENTS}

In this section, we solve equations (2-4) in a situation of practical interest for dimensions $n=4$ and $n=5$. Numerical values of the parameters are taken as follows: $L=100, R_{2}^{0}=\ldots=R_{n}^{0}=0.99, R_{1}^{L}=\ldots=R_{n-1}^{L}=$ 0.99 and $R_{n}^{L}=R_{\text {out }}=0.1$. The matrix $G$ of Raman gains is taken (up to terms of order $10^{-9}$ ) as

$$
G=10^{-3}\left[\begin{array}{ccccc}
0 & -5.354693 & -0.833641 & -0.165746 & -0.001215 \\
5.109551 & 0 & -5.091333 & -0.800871 & -0.246770 \\
0.757437 & 4.847864 & 0 & -4.883841 & -0.694188 \\
0.143011 & 0.724173 & 4.637914 & 0 & -3.546259 \\
0.001000 & 0.212878 & 0.628922 & 3.383213 & 0
\end{array}\right]
$$



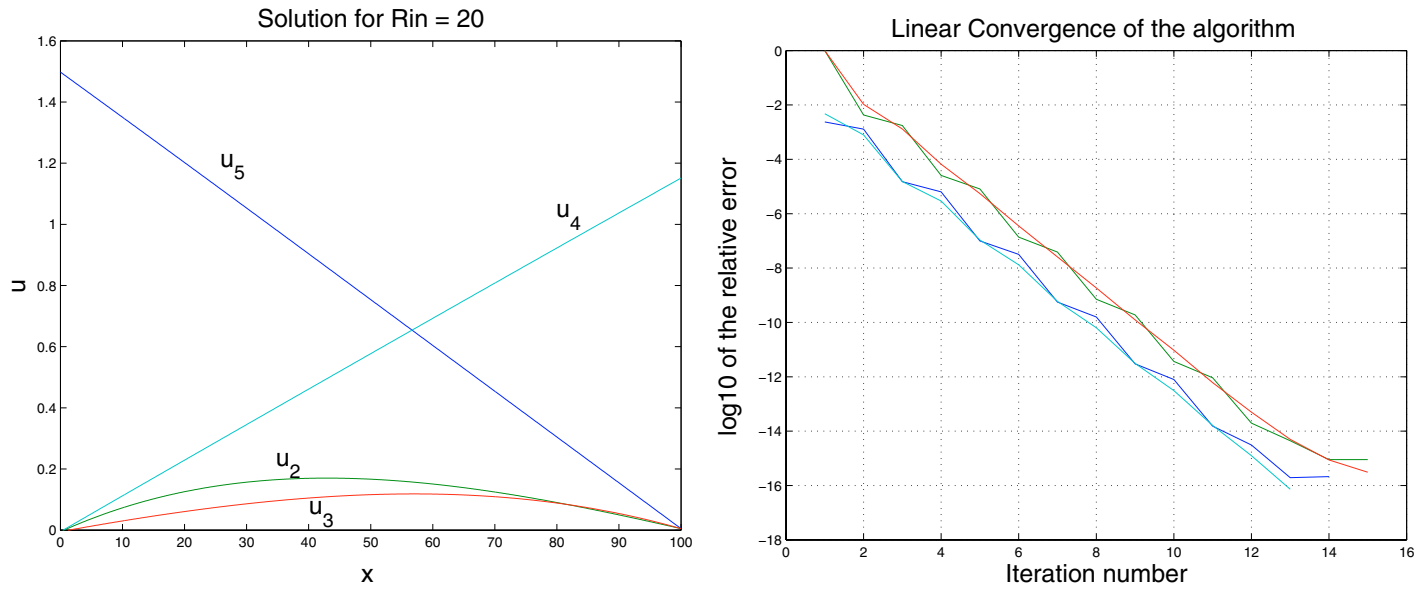

Figure 1. Solution $(F, B)$ for $n=4$ and relative error versus iteration number.

for $n=5$ and as the sub-matrix obtained by erasing the last column and the last line for $n=4$. Similarly, the matrix of attenuation coefficients is taken as the sub-matrix of appropriate size of

$$
\operatorname{diag}(\alpha)=10^{-3}\left[\begin{array}{ccccc}
0.388799 & 0 & 0 & 0 & 0 \\
0 & 0.346712 & 0 & 0 & 0 \\
0 & 0 & 0.296873 & 0 & 0 \\
0 & 0 & 0 & 0.252234 & 0 \\
0 & 0 & 0 & 0 & 0.218211
\end{array}\right]
$$

Note (see $[1,2,8]$ ) that the matrix $G$ is of the form

$$
G=L-D L^{T} D^{-1}
$$

where $L$ is a strictly lower triangular matrix and $D$ is a diagonal matrix with diagonal coefficients $\nu_{i}>0$ corresponding to $F_{i}$ and $B_{i}{ }^{1}$. In particular, $G D$ is skew-symmetric, and thus $G$ has always a non trivial kernel whenever $n$ is odd.

Algorithm (34) with explicit Euler as basic integration method and step-size $h=L / 1000$ has been used to solve equations (18-20) in the following three cases:

- $n=4$ and $R_{\text {in }}=20$ : a direct computation shows that the condition $q_{i}>0$ is fulfilled for $i=1, \ldots, 4$. The solution is plot on the left of Figure 1. The relative error on the solution $u$ in $\mathrm{L}^{1}$ is plot on the right of Figure 1 as a function of the number of iteration performed. A clear linear convergence is observed for this value of $R_{\mathrm{in}}$ as well as for larger ones as further numerical experiments confirm it.

- $n=4$ and $P=5$ : the fixed-point of the function $g$ as defined in (36) is searched through a fixed-point loop with initial value $R_{\text {in }}=20$. The solution $(F, B)$ is plot on the left of Figure 2. The fixed point is $R_{\text {in }} \simeq 43.708504$.

\footnotetext{
${ }^{1}$ This means that $G_{i j}=L_{i j}$ for $i>j$ and $G_{i j}=-L_{j i} \frac{\nu_{i}}{\nu_{j}}$ for $i<j$.
} 

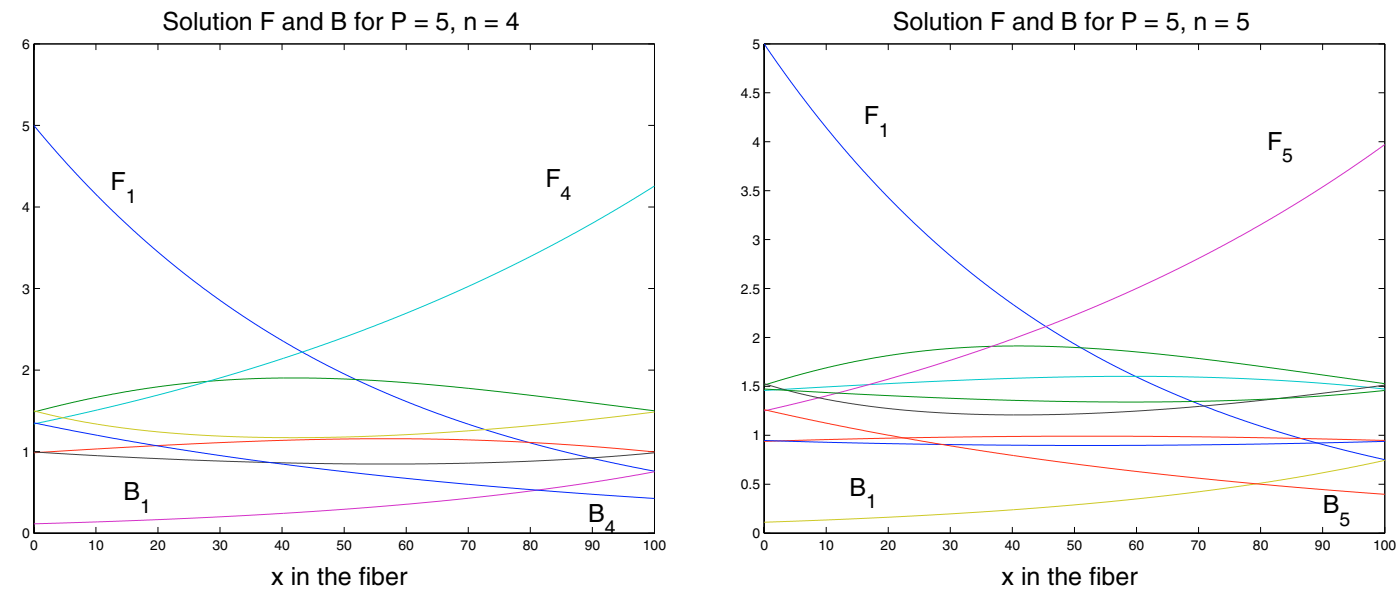

Figure 2. Solutions $(F, B)$ for $n=4, P=5$ (left) and $n=5$ (right).

- $n=5$ : the $a$ and $b$ vectors of Proposition 7.1 have the following values

$$
a \simeq\left[\begin{array}{c}
1 \\
-0.152781 \\
1.066044 \\
-0.198392 \\
1.551714
\end{array}\right], \quad b \simeq\left[\begin{array}{c}
1 \\
-0.145786 \\
0.968595 \\
-0.171179 \\
1.277317
\end{array}\right], \quad \text { and } \quad q^{0} \simeq 10^{2}\left[\begin{array}{c}
0.149596 \\
3.435993 \\
-0.465061 \\
3.352517 \\
0
\end{array}\right] .
$$

The solution $(F, B)$ is plot on the right of Figure 2. In this case, the sequence $\lambda^{(k+1)}=g\left(\lambda^{(k)}\right)(g$ as in (48)) converges to the numerical value $\lambda \simeq 242.0841567$.

\section{REFERENCES}

[1] M. Achtenhagen, T. Chang and B. Nyman, Analysis of a multiple-pump Raman amplifiers. Appl. Phys. Lett. 78 (2000) $1322-1324$.

[2] U. Ascher, R. Mattheij and R. Russel, Numerical Solution of Boundary Value for Ordinary Differential Equations. Prentice Hall, Englewood Cliffs (1988).

[3] F. Castella, P. Chartier and E. Faou, Analysis of a Poisson system with boundary conditions. C. R. Acad. Sci. Paris, Sér. I 336 (2003) 703-708.

[4] G. Golub and C. Van Loan, Matrix Computations. The Johns Hopkins University Press (1989).

[5] E. Hairer, C. Lubich, and G. Wanner, Geometric numerical integration Springer-Verlag 31 Springer Ser. Comput. Math. Berlin (2002). Structure-preserving algorithms for ordinary differential equations.

[6] H. Kidorf, K. Rottwitt, M. Nissov, M. Ma and E. Rabarijaona, Pump Interactions in a 100-nm Bandwidth Raman Amplifier. IEEE Photonics Technology Letters 11 (1999) 530-532.

[7] J. Pocholle, M. Papuchon, J. Raffy and E. Desurvire, Non linearities and optical amplification in single mode fibers. Revue Technique Thomson-CSF 22 (1990) 187-268.

[8] M. Rini, I. Christiani and V. Degiorgio, Numerical modeling and optimization of cascaded Raman fiber lasers. IEEE Journal of Quantum Electronics 36 (2000) 1117-1122. 